Verhindert man in einer anderen Versuchsdurchführung in einem Thermostaten bei $95^{\circ} \mathrm{C}$ jegliche Temperaturunterschiede und damit auch jegliche Konvektion, dann tritt auch nach Tagen keine Ätzkanalbildung ein. Andererseits kann auch bei Zimmertemperatur eine Ätzkanalbildung erzwungen werden, wenn man konzentrierte NaCl-Lösung langsam auf einen Steinsalzkristall auffließen läßt, worin man eine Bestätigung der obigen Schlußfolgerung sehen kann.

Ein weiterer Versuch weist auf die Wirkung eines Grenzflächentransportes bei der Ausspülung der Ätzkanäle hin. Legt man einen Kristall ein Jahr lang in reines Xylol, das noch $0,05 \%$ Wasser enthält, dann bilden sich bei geringer Heizung des Gefäßbodens ebenfalls Ätzkanäle, und zwar bezeichnenderweise jeweils in den Winkeln von Spaltstufen, die die Ausbildung von Konvektionswirbeln veranlassen.

Zusammenfassend kann also gesagt werden, daß auch völlig

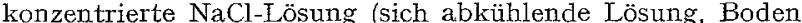
satz, Verdunstung von Lösungsmittel) einen Steinsalzkristal anätzen kann, wenn man dafür sorgt, daß eine Strömung in der Lösung vorhanden ist. Als Mechanismus wird ein durch Konvektion aktivierter Grenzflächentransport vermutet.

Ähnliche Betrachtungen lassen vielleicht die Probleme, die uns die Natur im Schriftgranit noch darbietet, in einem neuen Licht erscheinen.

Der Deutschen Forschungsgemeinschaft gilt mein Dank für Förderung und Unterstützung.

Eine ausführliche Veröffentlichung ist in der Zeitschrift für Kristallographie geplant.

Physikalisches Labor der Medizinischen Forschungsanstalt der Max-Planck-Gesellschafl, Göttingen

Eingegangen am 18. Jantuar 1964

WALTER GORSTI

1) Gerlach, He.: Diss. Hamburg, 1958.

\section{Ein solarer Hemisphäreneffekt in den erdmagnetischen Störungen}

Da die Sonnenflecken die unmittelbare Nachbarschaft des Sonnenäquators gewöhnlich meiden und in ihrer großen Mehrzahl heliographische Breiten zwischen 10 und $20^{\circ}$ bevorzugen, lag die Vermutung nahe, daß erdmagnetische Störungen in der Umgebung der Daten (5. März und 7. September), an denen die heliozentrische Breite $B_{0}$ der Erde ihre Extremwerte $\left( \pm 7^{\circ} 15^{\prime}\right)$ erreicht, verstärkt auftreten. Tatsächlich weisen die erdmagnetischen Störungen eine jährliche Doppelwelle mit Maxima in den Monaten März und September auf; aber schon vor längerer Zeit hat BARTELs ${ }^{1 a}$ ) Argumente dafür vorgebracht, daß diese jährliche Doppelwelle nicht als solarer Hemisphäreneffekt aufzufassen ist. Auch die neuesten erdmagnetischen Daten weisen in die gleiche Richtung; nach ihnen liegen die Maxima der Doppelwelle den Äquinoktien viel näher als den Daten höchster nördlicher und südlicher heliozentrischer Breite $B_{0}$ der Erde ${ }^{1 b}$ ).

Obwohl also die jährliche Doppelwelle der erdmagnetischen Störungen ihre Entstehung nicht der Breitenverteilung der Sonnenflecken verdankt, steckt in den erdmagnetischen Störungen doch ein solarer Hemisphäreneffekt, der von der ungleichen Verteilung der Sonnenflecken auf die beiden Sonnenhalbkugeln herrührt. Auf diesen Effekt hatte ich schon früher aufmerksam gemach $t^{2}$ ), ohne jedoch seine quantitative $\mathrm{Be}$ ziehung zur Nord-Süd-Asymmetrie der Sonnenfleckenverteilung zu präzisieren. Dies hole ich jetzt nach und kann damit die Existenz dieses Effekts überzeugend nachweisen. Dabei gehe ich folgendermaßen vor:

Wie aus der Tabelle ersichtlich, teile ich den Zeitraum 1889 bis 1953, für den sowohl die erdmagnetischen als auch die cum Vergleich nötigen solaren Daten vorliegen, nach den in ihm abgelaufenen Sonnenfleckenzyklen Nr. 13 bis 18 in sechs Abschnitte, bestimme dann innerhalb jedes dieser Abschnitte aus den erdmagnetischen Charakterzahlen ${ }^{3}$ ) den Mittelwert $C_{N}$ der täglichen erdmagnetischen Störungen für die Zeiträume, in denen die heliozentrische Breite $B_{0}$ der Erde die Bedingung $B_{0}>+5^{\circ}$ erfüllt, and den entsprechenden Mittelwert $C_{S}$ für $B_{0}<-5^{\circ}$, ermittle ferner innerhalb jedes der sechs Abschnitte der Tabelle aus den Greenwicher Daten ${ }^{4}$ ) die mittlere tägliche Fleckenfläche $F_{N}$ für die nördliche und $F_{S}$ für die südliche Sonnenhalbkugel und berechne schließlich aus den so gewonnenen Werten $C_{N}, C_{S}, F_{N}$ und $F_{S}$ die Nord-Suid-Asymmetrie $A_{u}$ im solaren Hemisphäreneffekt der erdmagnetischen Störungen und die Nord-Süd-Asymmetrie $A_{f}$ in der Verteilung der Sonnenflecken auf die beiden Hemisphären nach den Formeln

$A_{z \vartheta}=\left(C_{N}-C_{S}\right) /\left(C_{N}+C_{S}\right)$ und $A_{f}=\left(F_{N}-F_{S}\right) /\left(F_{N}+F_{S}\right)$;

Naturwissenschaften 1964
Tabelle. Die Nord-Süd-Asymmetrien im solaren Hemisphäreneffekt der erdmagnetischen Störungen und in der Verteilung der Sonnenflecken auf die beiden Hemisphären

\begin{tabular}{c|c|c|c}
\hline Zyklus Nr. & Jahre & $A_{w}$ & $A_{J}$ \\
\hline 13 & $1889-1900$ & $-0,051$ & $-0,110$ \\
14 & $1901-1912$ & $-0,002$ & $+0,027$ \\
15 & $1913-1922$ & $+0,019$ & $+0,097$ \\
16 & $1923-1932$ & $+0,010$ & $+0,079$ \\
17 & $1933-1943$ & $-0,030$ & $+0,012$ \\
18 & $1944-1953$ & $-0,010$ & $+0,032$
\end{tabular}

in dieser Weise erhalte ich die in der Tabelle mitgeteilten Werte von $A_{w}$ und $A_{f}$. Wie man sieht, ändern sich $A_{w}$ und $A_{f}$ von Zyklus zu Zyklus stets in gleichem Sinne; jedoch sind die Werte $A_{v,}$ gegenüber den Werten $A_{f}$ systematisch nach dem. Negativen hin, also zugunsten der Südhalbkugel der Sonne verlagert. Hierin kann sich ein Entfernungseffekt äußern, da die Erde während des Zeitraums $B_{0}>+5^{\circ}$ durchschnittlich weiter von der Sonne entfernt ist als während des Zeitraums $B_{0}<-5^{\circ}$. Die in den Zahlen der Tabelle zum Ausdruck kommende Korrelation zwischen $A_{w}$ und $A_{f}$ läßt sich durch die Gleichung

wiedergeben.

$$
A_{\vartheta}=\frac{1}{3} A_{f}-0,018
$$

Die Übereinstimmung in dem. Verlauf von $A_{q}$ und $A_{T}$ läßt keinen $Z$ weifel daran bestehen, daß es sich bei der Differenz zwischen $C_{N}$ und $C_{S}$ tatsächlich um einen solaren Hemisphäreneffekt handelt. Ausführlichere Angaben über diesen Effekt finden sich in $\mathrm{Nr}$. 8 der , Veröffentlichungen des Astronomischen. Instituts der Universität Frankfurt (Main)".

Astronomisches Institut der Universität, Frankfurt (Main)

Eingegangen am 8. Februar 1964

W. GLeissberg

1) Bartels, J.: a) Terrestrial Magnetism and Atmospheric Elec. 37, 1 (1932), sect. 9; - b) Ann. géophys. 19, 1 (1963), sect. 7. 2) GLEISSBERG, W.: a) Naturwissenschaften 44, 176 (1957); b) Z. Astrophysik 43, 144 (1957). - 3) Bartels, J.: a) Abhandl. Akad. Wiss. Göttingen, Math.-physik. K1., Sonderheft (1951); b) IAGA Bull. No. 18 (1962). — 4) Royal Greenwich Observatory: Sunspot and Geomagnetic-Storm Data 1874-1954. London 1955.

Zum Festhaften der mit Hydroxidionen beladenen, stark basischen Anionenaustauscherharzkugeln an der Glasoberfläche

Im AnschluB an die Harztüpfelmethode ${ }^{1}$ ) wurde bemerkt, daß die mit Hydroxidionen beladenen, stark basischen Anionenaustauscherharzkugeln in der wäßrigen Suspension an der Wand des Glasgefäßes so fest anhaften, wie es beim Harztüpfel-1) oder Harzflotationsverfahren ${ }^{2}$ ) nachteilig ist. Läßt man die wäßrige Suspension der mit Hydroxidionen neu beladenen Anionenaustauscherkügelchen wie Dowex 1 bzw. 2 auf die Glaswand langsam fließen, so bildet sich an der Wand eine dicht gepackte einheitliche Schicht der Harzkügelchen. Diese bildet sich aber nicht mehr als die 1 . Schicht bzw. die sog. "monolayer" der Kügelchen an der Glasoberfläche und haftet daran so fest an, daß sie auch durch heftiges Ausspritzen mit entionisiertem bzw. destilliertem Wasser nicht einfach weggenommen wird. Beim Eintropfen einer verdünnten wäßrigen Lösung eines starken Elektrolyts wie Natriumchlorid bzw. Ammoniumrhodanid oder Salzsäure wird diese Harzschicht dagegen sehr leicht ausgespült. Die Kationenaustauscherharze in der Säure- oder Salzform und die Anionenaustauscherharze in der Salzform haften an der Glasoberfläche nicht fest an. Man könnte daher schließen, daß es sich dabei um eine nahe wirkende topochemische Phasenassoziation zwischen der nassen Glasoberfläche und den auch an der Oberfläche der nassen stark basischen Austauscherkügelchen dicht vorhandenen und in deren unmittelbaren Nähe elektrolytisch stark dissoziierten Hydroxidionen ${ }^{3}$ ) handelt.

Laboratorium fiur analytische Chemie, Chemisches Institut dev naturwissenschaftlichen Fakultät der Universität, Tokyo, Japan

Masatoshi Fujimoto*)

Eingegangen am 26. Februar 1964

*) Zur Zeit Institut für Physikalische Chemie der Universität Tübingen; $a b$ 1.4.1964 Max-Planck-Institut für Physikalische Chemie, Göttingen.

1) Vgl. dazu z.B. Fujrmoto, M.: Chemist-Analyst 49, 4 (1960) bzw. Fujimoto, M., u. T. Iwamoto: Mikrochim. Acta 1963, 655.2) Fujлмото, M.: Naturwissenschaften 47, 252 (1960). - ${ }^{3}$ ) Hierzu 